\title{
Actividad física y su asociación con enfermedades crónicas en ancianos de once ciudades del Perú
}

\author{
Christian R. Mejia', Araseli Verastegui-Díaz², Dante M. Quiñones-Laveriano², \\ Germán Aranzabal-Alegría ${ }^{2}$ y Virgilio E. Failoc-Rojas ${ }^{3}$
}

${ }^{1}$ Escuela de Medicina Humana, Universidad Continental, Huancayo; Escuela de Postgrado, Universidad Privada Antenor Orrego, Trujillo; ${ }^{2}$ Instituto de Investigación en Ciencias Biomédicas, Facultad de Medicina Humana, Universidad Ricardo Palma, Lima; ${ }^{3}$ Facultad de Medicina Humana, Universidad Nacional Pedro Ruiz Gallo, Lambayeque. Perú

\begin{abstract}
Resumen
Objetivo: Determinar la frecuencia de actividad física y su asociación con enfermedades crónicas en ancianos de once ciudades del Perú. Métodos: Se realizó un estudio transversal analítico de datos secundarios. La variable dependiente fue la no realización de actividad física, y las independientes fueron las enfermedades crónicas y otras variables fisioantropométricas. Para el análisis bivariado y multivariado se usaron los modelos lineales generalizados. Se consideró un nivel de confianza del 95\% y un valor de $p<0.05$ como estadísticamente significativo. Resultados: De los 1,030 ancianos, el 68\% (698) no realizaba actividad física. Con el modelo multivariado se obtuvo que, por cada año de edad, aumentaba un 1.5\% la frecuencia no hacer actividad física (razón de prevalencia ajustada [RPa]: 1.015; intervalo de confianza del 95\% [IC 95\%]: 1.006-1.024; $p=0.001$ ); por cada centímetro de cintura adicional, disminuía un 0.6\% la realización de actividad física (RPa: 0.994; IC 95\%: 0.992-0.997; $p<0.001$ ); $y$ aquellos que padecían otra enfermedad cardiaca crónica (RPa: 1.15; IC 95\%:1.02-1.30; $p=0.019$ ) tenían mayores frecuencias de no realizar actividad física, ajustado por el sexo y por la ciudad de residencia. Conclusión: El no realizar actividad física se asoció con padecer alguna enfermedad cardiaca, mayor edad y el perímetro de cintura. Es necesario tener en cuenta estos resultados para promover la actividad física en esta población.
\end{abstract}

PALABRAS CLAVE: Anciano. Actividad motora. Enfermedades crónicas.

\begin{abstract}
Aim: To determine the frequency of physical activity and its association with chronic diseases in the elderly in eleven cities of Peru. Methods: A cross-sectional study of secondary data was conducted; the dependent variable was not the realization of physical activity, chronic disease and other physio-anthropometric variables were independent variables. For bivariate and multivariate analysis generalized linear models were used. A confidence level of $95 \%$ and $p<0.05$ as statistically significant was considered. Results: Of the 1030 elderly 68\% (698) did not perform physical activity. When performing the multivariate model was obtained for every year of age increased by $1.5 \%$ frequency do physical activity (RPA: 1.015, 95\% Cl 1.006-1.024, $p=0.001$ ) for each additional centimeter waist decreased 0.6\% performing physical activity (RPA: 0.994, 95\% Cl: 0.992-0.997, $p<0.001)$ and those suffering from other chronic heart disease (RPA: 1.15, 95\% Cl: 1.02-1.30. $p=0.019$ ) had higher frequencies of no physical activity, adjusted for sex and city of residence. Conclusion: No physical activity was associated with suffering from some heart disease, older age and waist circumference. We need to consider these results from the study to promote physical activity in this population.
\end{abstract}

KEY WORDS: Aged. Motor activity. Chronic disease.

\section{Correspondencia:}

Christian R. Mejía

Av. Las Palmeras, 5713

Lima 39, Perú

Fecha de recepción en versión modificada: 11-06-2016

E-mail: christian.mejia.md@gmail.com

Fecha de aceptación: 20-06-2016

DOI:10.24875/GMM.17002586

Gac Med Mex. 2017;153:480-485

Contents available at PubMed

www.gacetamedicademexico.com 


\section{Introducción}

El envejecimiento poblacional es una característica demográfica del siglo XXI, consecuencia de la mejora de las condiciones de vida, pero que a su vez va acompañado de un predominio de las enfermedades crónicas no transmisibles en edades más avanzadas'. Según la Organización Mundial de la Salud en su informe La situación mundial de las enfermedades no transmisibles (2014), estas son la principal causa de muerte en el mundo, con un $68 \%$ de defunciones en el año 2012 y afectando sobre todo a los países de ingresos medios y bajos². En Latinoamérica, el proceso de envejecimiento se caracteriza por su acelerado ritmo, en comparación con los países desarrollados 3 ; en el Perú, el último censo nacional revela que el $38.3 \%$ de los hogares tienen al menos una persona con más de 60 años de edad, y de esta población, el $71.4 \%$ de los varones y el $80.2 \%$ de las mujeres presenta algún problema de salud crónico, siendo las mujeres del área rural las que evidencian mayores deterioros de la salud, en comparación con las que viven en Lima Metropolitana 4 .

Aunque la actividad física no pueda detener el proceso biológico normal del envejecimiento, existe evidencia de que puede reducir la progresión de las enfermedades crónicas y minimizar los efectos del sedentarismo ${ }^{5,6}$. Según el Colegio Americano de Medicina Deportiva y la Asociación Americana del Corazón, los adultos mayores deben hacer por lo menos 30 minutos diarios de actividad física moderada a vigorosa, consistiendo en actividades recreativas o de ocio, desplazamientos o actividades ocupacionales (en caso de seguir laborando), entre otras ${ }^{3,7,8}$. Es por ello que aproximadamente 3,2 millones de defunciones se producen cada año como consecuencia de no realizar actividad física; más aún, para este grupo de edad, la importancia de la actividad física regular radica en que puede disminuir el riesgo de mortalidad por enfermedades cardiovasculares, diabetes tipo II, obesidad, cáncer, demencia y depresión $^{2,9-12}$, a partir de actividades de baja intensidad, como caminar. Además, los estudios que hablan de la actividad física en los ancianos confirman que aquellos que son físicamente activos tienen una menor probabilidad de experimentar alguna limitación funcional en comparación con los que son sedentarios $^{12,13}$.
Es por lo anteriormente mencionado que el objetivo de nuestro estudio fue determinar la frecuencia de la actividad física y su asociación con enfermedades crónicas en ancianos de once ciudades del Perú.

\section{Métodos}

Se realizó un estudio de tipo transversal analítico, derivado de un análisis secundario de datos. Se analizó la base perteneciente a la investigación que tuvo como objetivo principal evaluar la asociación entre la hipertensión arterial y la altitud de residencia en el Perú.

Los datos se obtuvieron de las encuestas realizadas a pacientes que acudieron al consultorio externo de cardiología de establecimientos de salud (hospitales públicos) de las siguientes ciudades del Perú: Piura, Chiclayo, Lima, Huancayo, Loreto, Cajamarca, Huánuco, Cusco, Puno, Cerro de Pasco y la Rinconada-Puno (Tabla 1). El tipo de muestreo fue no aleatorio. Se incluyeron los datos de los pacientes ancianos que hubieran respondido a la variable principal del estudio (no realizar actividad física).

La variable principal fue considerada según el no tener actividad física regular o según el tener un mínimo de 30 minutos diarios tres veces a la semana (categorías de respuesta: sí/no). Para la búsqueda de factores asociados a la realización de actividad física también se consideraron las variables de sexo (masculino/femenino), edad (considerada como variable cuantitativa para el análisis) y fisioantropométricas (presión arterial sistólica/diastólica, índice de masa corporal y diámetro de la cintura, todos considerados como variables cuantitativas), así como el padecimiento de enfermedades crónicas diagnosticadas médicamente (hipertensión arterial, diabetes mellitus, dislipidemia y alguna otra enfermedad cardiaca).

El protocolo fue aceptado por el comité de ética del Hospital Nacional San Bartolomé (OFICIO No: 2845-DG-OADI-N ${ }^{\circ}$ 822). Luego de la recepción de la base de datos se procedió al control de calidad de los mismos; esto fue realizado por dos autores de forma separada (que verificaron los datos según los que se tenían en las encuestas), que juntaron en una misma matriz (posterior a comprobar que los datos coincidían). Todo esto se realizó en una hoja de datos del programa Excel (versión 2010 para Windows). Se 
Tabla 1. Características demográficas de la población

\begin{tabular}{|c|c|c|c|c|}
\hline Ciudad & $\begin{array}{l}\text { Región } \\
\text { natural* }\end{array}$ & $\begin{array}{l}\text { Población } \\
\text { total* }\end{array}$ & $\begin{array}{c}\text { Altitud media } \\
\text { (metros sobre } \\
\text { nivel mar)* }\end{array}$ & $\begin{array}{l}\text { Nivel } \\
\text { socioeconómico }\end{array}$ \\
\hline Piura & Costa & $1,784,551$ & 29 & Regular \\
\hline Chiclayo & Costa & 716,732 & 27 & Regular \\
\hline Lima & Costa & $9,752,000$ & 154 & Aceptable \\
\hline Huancayo & Sierra & 323,054 & 3271 & Regular \\
\hline Cajamarca & Sierra & 162,326 & 2750 & Pobre \\
\hline Huánuco & Sierra & 149,210 & 1800 & Pobre \\
\hline Cusco & Sierra & 348,935 & 3390 & Regular \\
\hline Puno & Sierra & 120,229 & 3815 & Pobre \\
\hline Cerro de Pasco & Sierra & 70,000 & 4330 & Muy pobre \\
\hline $\begin{array}{l}\text { La } \\
\text { Rinconada-Puno }\end{array}$ & Sierra & 50,000 & 5100 & Extrema pobreza \\
\hline
\end{tabular}

realizó el análisis estadístico con el programa Stata versión 11.1 (Stata Corp, Texas, USA). Para la descripción de las variables numéricas se determinó su normalidad con la prueba estadística Shapiro Wilk, con la mediana y los rangos intercuartílicos. Para la descripción de las variables categóricas se usaron las frecuencias y los porcentajes.

Para el análisis bivariado se obtuvieron los valores de $p$, las razones de prevalencias crudas y ajustadas (RPa), y los intervalos de confianza al 95\% (IC 95\%). Se usaron modelos lineales generalizados, con la familia Poisson y la función de enlace log, y además se tomó en cuenta la singularidad de las poblaciones encuestadas; se usó como cluster la sede de encuestado. Se consideró un nivel de confianza del 95\% y un valor de $p<0.05$ como estadísticamente significativo.

\section{Resultados}

De los 1030 encuestados, el 52.7\% (543) fueron del sexo femenino y la mediana de edad fue de 68 años (rango intercuartílico: 64-74 años). El 32.8\% (338) padecía hipertensión arterial, el 12.7\% (130) diabetes mellitus, el $23.3 \%$ (238) dislipidemia y el $13.0 \%$ (133) alguna otra enfermedad cardiaca crónica. Los otros valores descriptivos se muestran en la tabla 2.
Tabla 2. Características fisiopatológicas de los ancianos encuestados en 11 ciudades del Perú

\begin{tabular}{lcc}
\hline Variable & $\mathbf{n}$ & $\%$ \\
\hline Sexo & & \\
$\quad$ Masculino & 52.7 & 543 \\
$\quad$ Femenino & 47.3 & 487 \\
Edad (años) ${ }^{*}$ & 68 & $64-74$ \\
Presión sistólica $(\mathrm{mmHg})^{*}$ & 120 & $110-130$ \\
Presión diastólica $(\mathrm{mmHg})^{*}$ & 72.8 & $66-80$ \\
& & \\
Índice de masa corporal $\left(\mathrm{kg} / \mathrm{m}^{2}\right)^{*}$ & 26.3 & $23.8-29$ \\
& & \\
Diámetro de cintura $(\mathrm{cm})^{*}$ & 92 & $82-100$ \\
& & \\
Padece: & 338 & 32.8 \\
$\quad$ Hipertensión arterial & 130 & 12.7 \\
$\quad$ Diabetes mellitus & 238 & 23.3 \\
$\quad$ Dislipidemia & 133 & 13.0 \\
$\quad$ Otra enfermedad cardiaca crónica & & \\
${ }^{*}$ Mediana y rango intercuartilico. &
\end{tabular}

El $67.8 \%$ (698) de los ancianos manifestaron que no realizaba actividad física. Al realizar el análisis bivariado de esta variable con las secundarias se obtuvo que se asoció al sexo $(p=0.015)$, la edad ( $p=0.001)$, el diámetro de la cintura $(p<0.001)$ y padecer otra enfermedad cardiaca crónica $(p=0.014)$, como se muestra en la tabla 3.

Al realizar el modelo multivariado se obtuvo que, por cada año de edad, aumentaba un $1.5 \%$ la frecuencia 
Tabla 3. Análisis bivariado de la asociación de enfermedades crónicas y no realización de actividad física en ancianos de once ciudades del Perú

\begin{tabular}{|c|c|c|c|c|}
\hline \multirow[t]{2}{*}{ Variable } & \multicolumn{2}{|c|}{ Actividad física* } & \multirow[t]{2}{*}{ RPc (IC 95\%) } & \multirow[t]{2}{*}{$p$} \\
\hline & Sí n (\%) & No $\mathrm{n}(\%)$ & & \\
\hline \multicolumn{5}{|l|}{ Sexo } \\
\hline Femenino & $388(55.6)$ & $155(46.7)$ & $1.12(1.02-1.23)$ & 0.015 \\
\hline Masculino & $310(44.4)$ & $177(53.3)$ & & \\
\hline Edad (años) ${ }^{\dagger}$ & $68(64-75)$ & $66(62-72)$ & $1.016(1.011-1.021)$ & 0.001 \\
\hline Presión sistólica $(\mathrm{mmHg})^{+}$ & $120(110-130)$ & $120(105-130)$ & $1.003(0.999-1.007)$ & 0.185 \\
\hline Presión diastólica $(\mathrm{mmHg})^{\dagger}$ & $75(68-80)$ & $70(65-80)$ & $1.004(0.998-1.011)$ & 0.195 \\
\hline Índice de masa corporal $\left(\mathrm{kg} / \mathrm{m}^{2}\right)^{\dagger}$ & $26(23.7-28.9)$ & $26.5(23.9-29)$ & $0.996(0.989-1.003)$ & 0.295 \\
\hline Diámetro de cintura $(\mathrm{cm})^{\dagger}$ & $91(80.5-99)$ & $94(86-101)$ & $0.994(0.991-0.997)$ & $<0.001$ \\
\hline \multicolumn{5}{|l|}{ Padece: } \\
\hline Hipertensión arterial & $245(35.1)$ & $93(28.0)$ & $1.11(0.94-1.31)$ & 0.230 \\
\hline Diabetes mellitus & $100(14.4)$ & $30(9.1)$ & $1.16(0.94-1.42)$ & 0.172 \\
\hline Dislipidemia & $164(23.6)$ & $74(22.8)$ & $1.01(0.93-1.11)$ & 0.771 \\
\hline Otra enfermedad cardiaca crónica & $105(15.1)$ & $28(8.5)$ & $1.19(1.04-1.37)$ & 0.014 \\
\hline
\end{tabular}

*Considerada como tener actividad física regular un mínimo de 30 minutos diarios tres veces a la semana.

${ }^{\dagger}$ Mediana y rango intercuartílico.

IC 95\%: intervalo de confianza al 95\%; RPc: razón de prevalencias cruda.

IC 95\% y p obtenidos con modelos lineales generalizados, con familia Poisson, función de enlace log, modelos robustos y tomando en cuenta cada sede como grupo cluster.

de no hacer actividad física (RPa: 1.015; IC 95\%: 1.006-1.024; $p=0.001$ ); por cada centímetro de cintura adicional, disminuía un $0.6 \%$ la realización de actividad física (RPa: 0.994; IC 95\%: 0.992-0.997; $\mathrm{p}<0.001)$; y aquellos que padecían otra enfermedad cardiaca crónica (RPa: 1.15; IC 95\%: 1.02-1.30; $p=0.019)$ tenían mayores frecuencias de no realizar actividad física, ajustado por el sexo y por la ciudad de residencia.

\section{Discusión}

En la actualidad, la vida sedentaria se ha generalizado como una característica predominante, y concomitantemente el aumento de las enfermedades crónicas que constituyen la principal causa de muerte en todas las regiones del mundo ${ }^{14,15}$, a pesar de que se ha demostrado que el ejercicio físico previene la aparición de múltiples patologías y condiciones que traen consecuencias directas e indirectas para la sociedad $^{16}$.

Los hallazgos de nuestro estudio demuestran que la edad está relacionada con la realización de actividad física en el adulto mayor, lo que concuerda con lo encontrado por Berkemeyer, et al. ${ }^{17}$, quienes al realizar un estudio en el Reino Unido y los EE.UU., en una población de 4052 y 3459 adultos mayores con edades de 49-91 y 49-85 años, respectivamente, reportaron que la frecuencia y la intensidad de la actividad física cambian a lo largo de la vida, siendo significativamente menores a medida que va avanzando la edad ${ }^{17}$. Esto es ratificado por dos estudios locales que muestran que el incremento de la edad está asociado a un mayor riesgo de evento cardiovascular y al padecimiento de síndrome metabólico entre trabajadores peruanos ${ }^{18,19}$. Por lo tanto, el aumento de la edad se considera un factor de riesgo para el desarrollo y la progresión de la mayoría de las enfermedades crónicas, siendo la actividad física regular un modificador sustancial de estos riesgos ${ }^{5}$. Esto debe ser tomado muy en cuenta por los promotores de programas del adulto mayor y otros similares, para que puedan dirigir sus esfuerzos a los adultos de avanzada edad con programas hechos según sus posibilidades físicas, además de otras estrategias para incentivar la práctica de actividad física constante.

También se encontró que el diámetro de la cintura estaba asociado a realizar actividad física, lo que es concordante con el estudio de Villalobos y García ${ }^{20}$, que encontraron que a medida que avanza la edad disminuye la masa magra y aumenta la masa grasa, 
redistribuyéndose principalmente en la zona abdominal y constituyendo así la obesidad abdominal, que podría disminuir la capacidad de ejercicio. Además, la adiposidad central estaría en relación con un incremento en la morbimortalidad de diversas enfermedades crónicas, como la hipertensión, la enfermedad coronaria y la diabetes tipo $\mathrm{I}^{20}$.

Por último, los que tenían una enfermedad cardiaca crónica realizaban menor actividad física $(p=0.014)$. Espinosa, et al..$^{21}$ aplicaron durante 12 meses un programa de rehabilitación que incluía ejercicio físico en 113 pacientes con antecedente de infarto de miocardio de bajo riesgo, y observaron una mejoría de la calidad de vida y de la tolerancia al esfuerzo. Diversos estudios encuentran que la actividad física se asocia con una reducción del 35\% del riesgo de enfermedad cardiovascular en hombres y mujeres, y además se produjo una reducción del riesgo de enfermedades metabólicas y cardiorrespiratorias; estos beneficios podrían deberse en parte a que los ejercicios tienen efectos antiinflamatorios ${ }^{22,23}$. Otros estudios sobre la prescripción de ejercicio como prevención y terapia en enfermedades crónicas confirman que reduce la mortalidad cardiaca en un $26 \%$, además de disminuir el colesterol total, los triglicéridos, la glucemia, la obesidad, el estrés y la presión arterial sistólica, entre otros beneficios; e incluso parece recomendable introducir el ejercicio de resistencia en la rehabilitación de pacientes con insuficiencia cardiaca, teniendo en cuenta las particularidades de cada paciente ${ }^{24-26}$. Todo esto ratifica lo encontrado en el estudio, lo que puede aportar valores propios de nuestro medio para que posteriormente se realicen estudios de intervención o programas de prevención en esta población.

El estudio tuvo la limitación de que, al ser un análisis de datos secundarios, no se pudo tener acceso a otras variables importantes, como saber si estuvieron inscritos a algún programa de adulto mayor o el nivel de apoyo de la familia. Además, el sesgo de selección imposibilita el usar de manera certera los intervalos de confianza. A pesar de estas limitaciones, los resultados son importantes porque reflejan una realidad en una cantidad importante de población anciana de diversas ciudades del Perú.

Según los resultados encontrados, se concluye que no realizar actividad física en el adulto mayor se asocia con padecer alguna enfermedad cardiaca, mayor edad y el perímetro de cintura. Por ello es importante promover la realización de actividad física, a través de programas dirigidos a este grupo poblacional, para poder retardar o reducir significativamente el riesgo de aparición de enfermedades crónicas, pudiendo así disminuir la mortalidad.

\section{Conflictos de intereses}

Los investigadores declaran no tener ningún conflicto de intereses.

\section{Financiamiento}

La investigación fue autofinanciada.

\section{Bibliografía}

1. Rojas DV. Morbilidad y mortalidad del adulto mayor en un servicio de medicina de un hospital general del Perú. Rev Peru Epidemiol. 2010;14:99-107.

2. Mendis S, Armstrong T, Bettcher D, et al. Informe sobre la situación mundial de las enfermedades no transmisibles 2014. Cumplimiento de las nueve metas mundiales relativas a las enfermedades no transmisibles: una responsabilidad compartida. Organización Mundial de la Salud; 2014. p. 16. Disponible en: http://apps.who.int/iris/bitstream/10665/149296/1/WHO_NMH_NVI_15.1_spa.pdf?ua=1\&ua=1.

3. Diana C, Parra AH. Ambientes urbanos y actividad física en adultos mayores: relevancia del tema para América Latina. Rev Salud Pública. 2010;12:327-35.

4. Situación de la población adulta mayor. Perú: Instituto Nacional de Estadística y Demografía; 2015. p. 44. Report No.: 9. Disponible en: https:// www.inei.gob.pe/media/MenuRecursivo/boletines/informe-tecnico_poblacion-adulto-mayor-jul-ago-set2015.pdf.

5. American College of Sports Medicine, Chodzko-Zajko WJ, Proctor DN, Fiatarone Singh MA, et al. American College of Sports Medicine position stand. Exercise and physical activity for older adults. Med Sci Sports Exerc. 2009;41:1510-30.

6. Trachte F, Geyer S, Sperlich S. Impact of physical activity on self-rated health in older people: do the effects vary by socioeconomic status? J Public Health (Oxf). 2016;38:754-9.

7. Nelson ME, Rejeski WJ, Blair SN, et al. Physical activity and public health in older adults: recommendation from the American College of Sports Medicine and the American Heart Association. Circulation. 2007;116:1094-105.

8. Tuvemo Johnson S, Martin C, Anens E, et al. Older adults' opinions on fall prevention in relation to physical activity level. J Appl Gerontol. 2016:733464815624776.

9. Cerin E, Sit CHP, Zhang CJP, et al. Neighbourhood environment, physical activity, quality of life and depressive symptoms in Hong Kong older adults: a protocol for an observational study. BMJ Open. 2016;6: e010384.

10. Brandt $C$, Pedersen BK. The role of exercise-induced myokines in muscle homeostasis and the defense against chronic diseases. J Biomed Biotechnol. 2010;2010:520258.

11. Straight CR, Brady AO, Evans EM. Moderate-intensity physical activity is independently associated with lower-extremity muscle power in older women. Women Health. 2016;56:871-84.

12. O'Doherty MG, Cairns K, O'Neill V, et al. Effect of major lifestyle risk factors, independent and jointly, on life expectancy with and without cardiovascular disease: results from the Consortium on Health and Ageing Network of Cohorts in Europe and the United States (CHANCES). Eur J Epidemiol. 2016;64:1-14

13. Yorston LC, Kolt GS, Rosenkranz RR. Physical activity and physical function in older adults: the 45 and up study. J Am Geriatr Soc. 2012;60:719-25.

14. Ledón Llanes L. Enfermedades crónicas y vida cotidiana. Rev Cuba Salud Pública. 2011;37:488-99.

15. Archer E, Blair SN. Physical activity and the prevention of cardiovascular disease: from evolution to epidemiology. Prog Cardiovasc Dis. 2011:53:387-96.

16. Martín Lesende I, Gorroñogoitia Iturbe A, Gómez Pavón J, et al. El anciano frágil. Detección y tratamiento en AP. Aten Primaria. 2009;42:388-93. 
17. Berkemeyer K, Wijndaele K, White T, et al. The descriptive epidemiology of accelerometer-measured physical activity in older adults. Int Behav Nutr Phys. 2016;13:2.

18. Mejia CR, Espejo RP, Zevallos KR, et al. Factores asociados al riesgo cardiovascular según Framingham en taxistas de una empresa de Huancayo, Perú. Rev Asoc Esp Espec Med Trab. 2016;25:19-25.

19. Mejía CR, Quiñones-Laveriano DM, Cruzalegui-Solari CC, et al. Edad como factor de riesgo para desarrollar síndrome metabólico en trabajadores mineros a gran altura. Rev Argent Endocrinol Metab. 2016;53:29-35.

20. Villalobos Silva JA, García Zenón T. Malnutrición en el anciano. Parte II: obesidad, la nueva pandemia. Med Interna México. 2012;28:154-61.

21. Espinosa Caliani S, Bravo Navas JC, Gómez-Doblas JJ, et al. Rehabilitación cardíaca postinfarto de miocardio en enfermos de bajo riesgo.
Resultados de un programa de coordinación entre cardiología y atención primaria. Rev Esp Cardiol. 2004;57:53-9.

22. Handschin C, Spiegelman BM. The role of exercise and PGC1a in inflammation and chronic disease. Nature. 2008;454:463-9.

23. Gleeson M, Bishop NC, Stensel DJ, et al. The anti-inflammatory effects of exercise: mechanisms and implications for the prevention and treatment of disease. Nat Rev Immunol. 2011;11:607-15.

24. Pedersen BK, Saltin B. Evidence for prescribing exercise as therapy in chronic disease. Scand J Med Sci Sports. 2006;16(Supl 1): 3-63.

25. Boraita Pérez A. Ejercicio, piedra angular de la prevención cardiovascular. Rev Esp Cardiol. 2008;61:514-28.

26. Rivas-Estany E. El ejercicio físico en la prevención y la rehabilitación cardiovascular. Rev Esp Cardiol. 2011;11:18-22. 